Case Report

\title{
Treatment of Iliopsoas Tendinitis after a Left Total Hip Arthroplasty with Botulinum Toxin Type A
}

David E Fish, MD, and Wanda S. Chang, BS

From: David Geffen School of Medicine at UCLA, Los Angeles

Dr. Fish is Associate Clinical Professor, Department of Orthopaedics, Physical Medicine and Rehabilitation, David Geffen School of Medicine at UCLA.

Ms Chang is a fourth year medical student, David Geffen School of

Medicine at UCLA

Address correspondence: David E. Fish, MD

Assistant Professor, Dept. of Orthopaedics

Physical Medicine and Rehabiliation UCLA School of Medicine 1245 16th Street Tower Bldg 7th Floor,

$\mathrm{Rm} 715$

Santa Monica, CA 90404

E-mail: dfish@mednet.ucla.edu

Disclaimer: No external funding was received for this study. Conflict of interest: None

Manuscript received: 04/10/2007 Revisions received:06/10/2007 Accepted for Publication 06/21/2007

Free full manuscript: www.painphysicianjournal.com
Objective: Treatment of iliopsoas tendinitis after a left total hip arthroplasty with botulinum toxin type $A(B T X-A)$.

Design: Case study

Setting: Outpatient musculoskeletal clinic.

Participants: Single patient subject with left iliopsoas tendinitis after a left total hip arthroplasty.

Interventions: Fluoroscopically guided injection of 100 units of BTX-A into the left iliopsoas muscle.

Main Outcome Measures: Functional improvement with Oswestry Disability Index (ODI) and scores using a 0-10 point pain intensity numerical rating scale (PI-NRS) at one and 6 months follow up.

Results: A 71-year-old female with 4 months of left groin pain after successful total hip arthroplasty. Work up did not reveal any infection or malpositioning of the left hip prosthetic components. Attempts at aspiration revealed no fluid in the joint space. A positive xylocaine muscle block confirmed a diagnosis of iliopsoas tendinitis. The BTX-A injection resulted in an improved left groin pain rating from a baseline of 7 to 1 and improved ability to flex her left hip at one month follow up. Pain was rated 8 at six months follow up. ODI improved from a baseline of $26 \%$ to $22 \%$ at one month follow up and $18 \%$ at 6 months follow up.

Conclusions: lliopsoas tendinitis is an uncommon cause of groin pain but has been described after a total hip arthroplasty in several case reports. Non-operative management has not been well established but includes anesthetic and corticosteroid injection into the muscle. The use of botulinum toxin A provided significant pain relief, functional improvement, and may represent an alternative to the surgical management of iliopsoas tendinitis.

Key words: Rehabilitation, iliopsoas tendinitis, iliopsoas bursitis, total hip arthroplasty, groin pain, botulinum toxin.

Pain Physician 2007; 10:565-571 


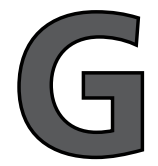

roin or anterior hip pain can be due to numerous causes. In the setting of a patient who has undergone a total hip arthroplasty (THA), the differential may include multiple causes ranging from infection; tumor; aseptic or septic loosening of the components, occult fracture; heterotopic ossification; referred pain from the spine, knee, retroperitoneum or abdomen; and soft tissue inflammation. Thought to be a rare cause of groin pain, there have been several case reports of iliopsoas tendinitis (1-5) and bursitis (6) causing significant pain in patients who underwent THA. One prospective study of 206 total hip arthroplasties found an incidence of $4.3 \%$ of patients experiencing pain after surgery (7). Iliopsoas tendinitis and bursitis are interrelated because of their close proximity such that inflammation of one may result in inflammation of the other (8).

We present a patient with persistent left groin pain one year after a left THA that responded well to a fluoroscopically guided botulinum toxin type $A$ (BTX-A) injection into her left iliopsoas muscle.

\section{Case Report}

A 71-year old woman presented to an outpatient musculoskeletal clinic with worsening, constant, left groin pain for the past 4 months exacerbated by walking, getting into and out of her car, and going up a flight of stairs. One year previous to presentation, she underwent an uncemented left total hip arthroplasty for end-stage osteoarthritis without any perioperative complications and initial resolution of her left hip pain. Her current pain was not relieved by oral analgesics or nonsteroidal medication. Physical examination revealed a normal spine examination but pain on palpation of the left hip flexors that worsened with resisted left hip flexion. There were no palpable masses or audible clunk over her left inguinal area. The patient had an antalgic gait without the use of an assistive device. Her coordination, sensation, and reflexes were intact and symmetric. She had a negative straight leg raise bilaterally. Muscle strength was 5/5 bilaterally and tone was normal. Her extremities had intact peripheral pulses and without clubbing, cyanosis, or edema.

Plain radiographs showed an anatomically aligned left total hip arthroplasty (Fig.1). A technetium bone

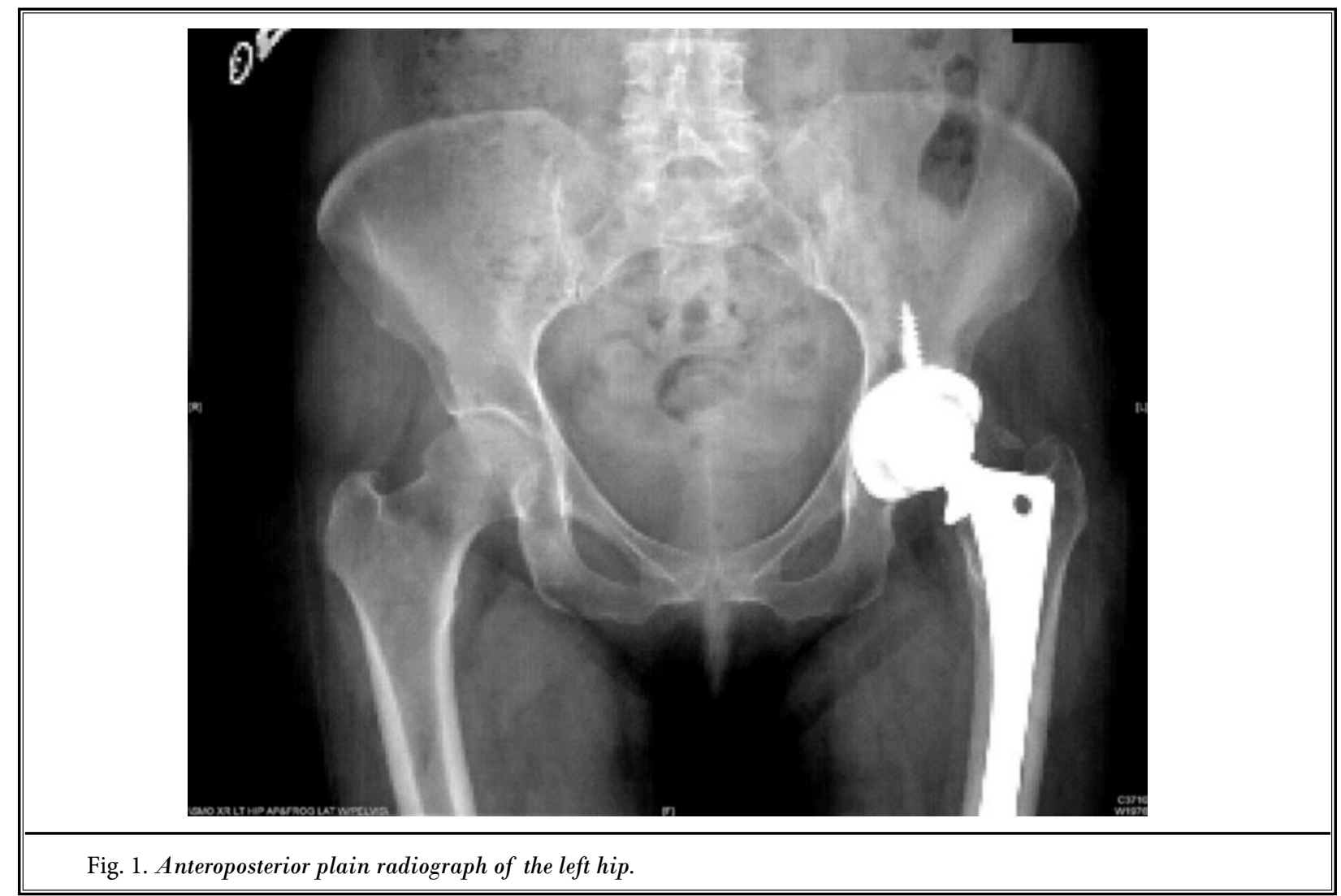


scan was negative for loosening or infection. An attempt to aspirate the left hip joint was negative for free fluid. The next step was to attempt a left iliopsoas muscle block under fluoroscopy. Using an anteriorposterior view (AP), a 23-gauge, 3.5-inch spinal needle was directed and advanced on the left in the inferior portion of the transverse process of the lumbar spine. Once this was done, the lateral view showed the position in the mid-point of the vertebral body. Once the needles were in a proper location at the level of the transverse process of $L 2, L 3$, and $L 4$, an injection with $2 \mathrm{ml}$ of contrast outlined the left iliopsoas muscle. At this point, the mixture for injection was $6 \mathrm{ml}$ of $1 \%$ Xylocaine, and one month later, the patient returned for a follow up and reported a transient but nearly complete relief of her groin pain. The patient then underwent a fluoroscopically guided left iliopsoas muscle injection using the same approach as mentioned above with 100 units of botulinum toxin A distributed over the same 3 sites (Fig. 2).

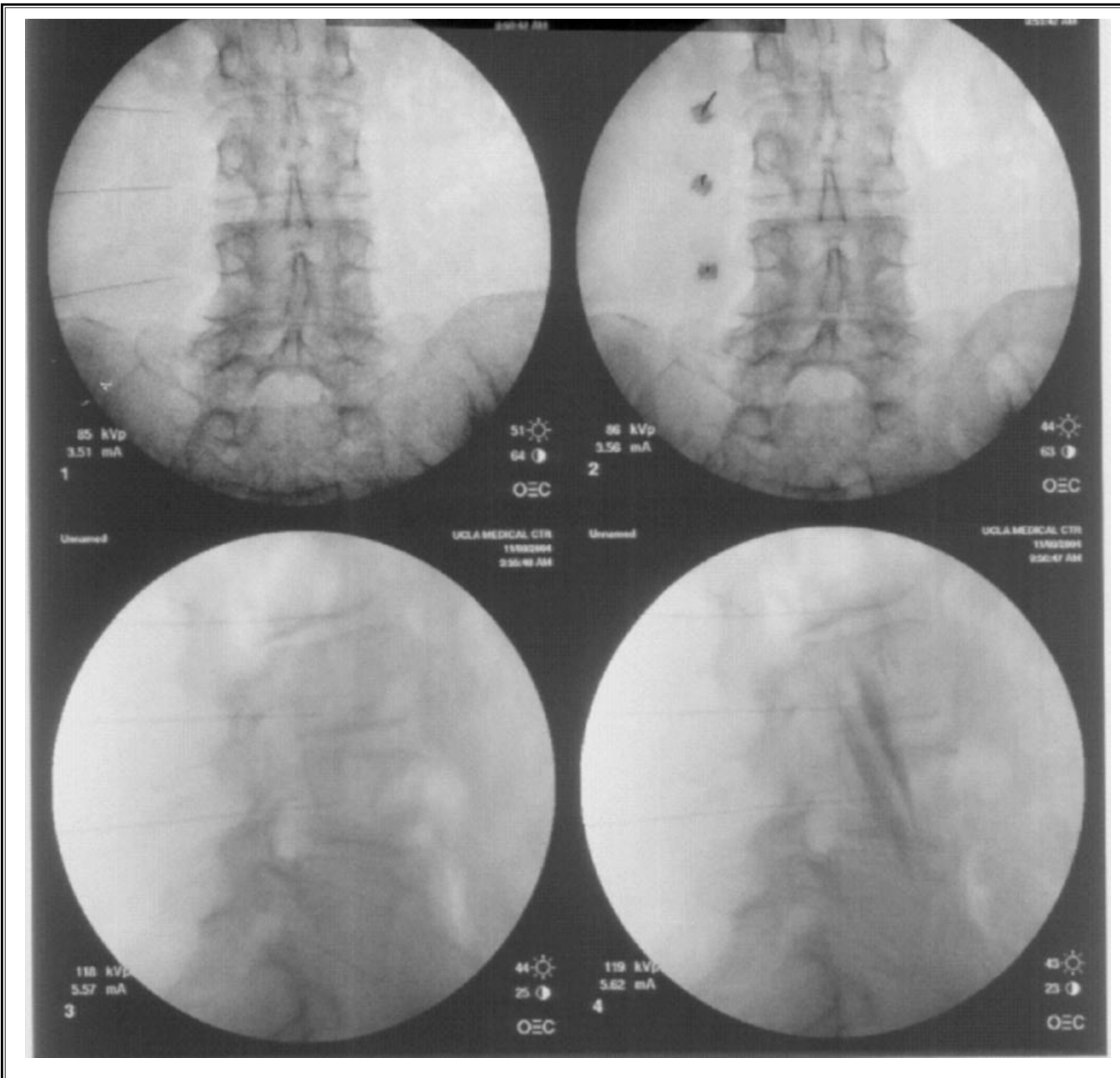

Fig. 2. Fluoroscopic views of injection to the left iliopsoas. 1. Marker positions for needle placement in anteroposterior view. 2. Proper anteroposterior needle placement. 3. Proper lateral needle placement. 4. Contrast spread showing iliopsoas muscle outline. 
Her Oswestry Disability Index (ODI) was calculated to be $26 \%$ before the BTX-A injection with a pain level of 7 out of 10 on a numerical pain scale. At the 1 month follow up, her ODI was $22 \%$, pain level was 1, and she had increased range of motion in left hip flexion. At the 6 months follow up, her ODI was $18 \%$ and pain level was 8 . The plan is to have the patient schedule for a repeat fluoroscopically guided injection of botulinum toxin $A$ to her left iliopsoas muscle.

\section{Discussion}

\section{Iliopsoas Tendonitis and Bursitis}

The psoas and iliacus muscle originate from the lumbar spine and pelvis and inserts as the tendon anteromedially to the lesser trochanter of the femur. The role of the iliopsoas is that of a hip flexor and femur external rotator (8). Tendinitis of the iliopsoas usually occurs at the junction of the muscle and tendon. Inflammation of the iliopsoas bursa, the largest in the body and located deep to the tendon (Fig. 3a), can occur alone or with associated tendinitis. The 3 main causes of iliopsoas bursitis are rheumatoid arthritis, acute trauma, and overuse injury. It has been postulated that acute or chronic occupational or sports activity have accounted for the majority of the cases reported where there is vigorous hip flexion and extension (8). One thought is with hip flexion, the iliopsoas muscle and anterior portion of the bursa moves away from the hip joint, but with hyperextension, the tension generated stretches the iliopsoas and bursa, which causes trauma (9). Another theory is that during extension of a flexed, abducted, and externally rotated hip, the normal lateral to medial motion of the iliopsoas tendon is interrupted resulting in a painful snapping of the tendon over the femoral head and anterior capsule (10). Both conditions can occur concurrently and are essentially identical in their clinical presentations (8).

The average time from onset of symptoms to diagnosis is 32 to 41 months as poor localization represents a challenge (8). Moreover, a thorough history and directed physical examination will help distinguish an inflamed iliopsoas muscle and/or bursa from the differential causes of anterior hip pain. A history of constant anterior hip pain aggravated by activity such as active flexion, ascending stairs, or flexing the hip to get out of a car or bed is strongly suggestive of the diagnosis; whereas, in septic or aseptic loos- ening, there is pain upon weight bearing. Tenderness may be felt on deep palpation over the musculotendinous junction (Fig. 3b) $(8,15)$. The most commonly

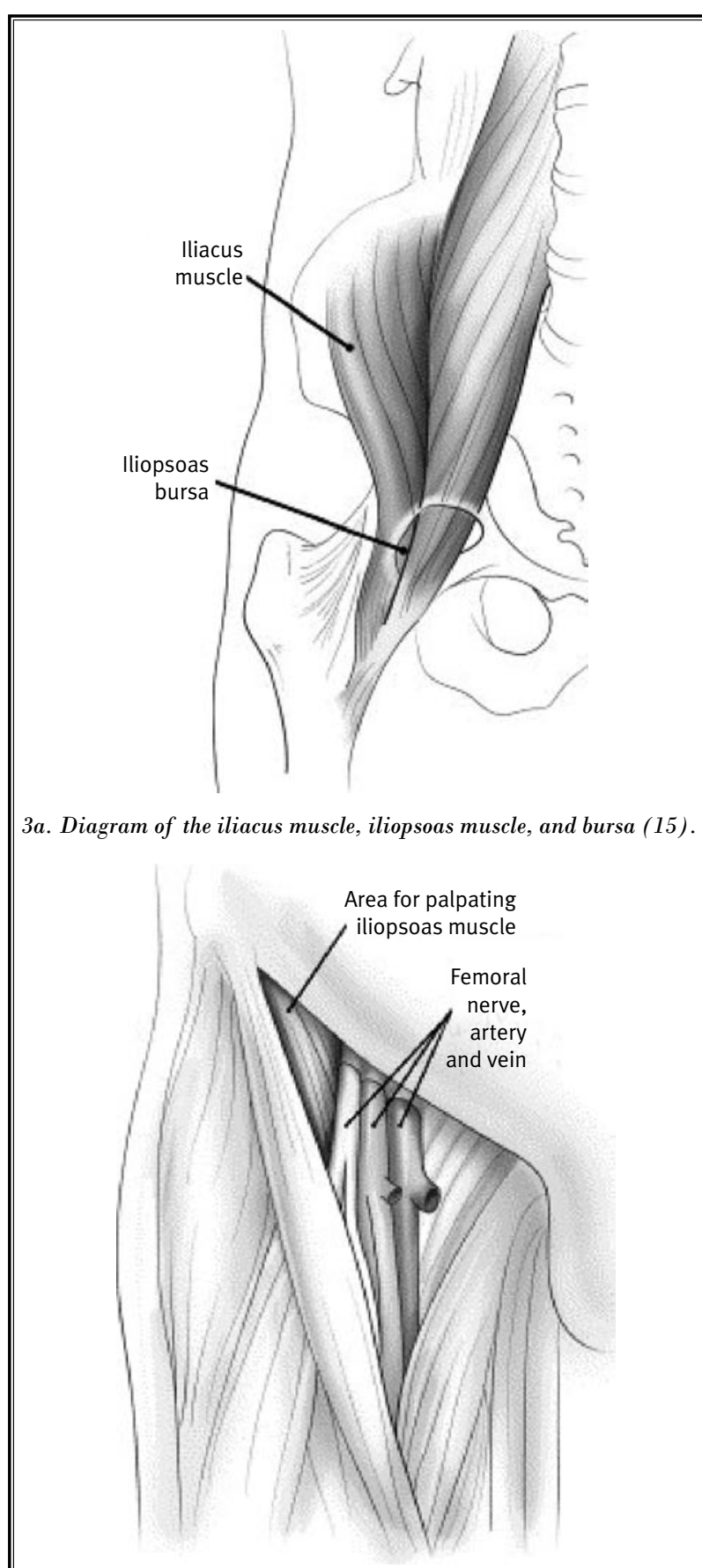

3b. Diagram of the area for palpating the iliopsoas muscle (15).

Fig. 3. Diagram of iliacus muscle, iliopsoas muscle, bursa and where to palpate. 
reported clinical maneuver is the "snapping hip sign" or "extension test" whereby pain with or without a palpable snap is reproducible by deep manual palpation of the inguinal crease while having the patient flex the hip then abduct and externally rotate the hip. Upon returning to the neutral position, the examiner palpates for a click (8). Another helpful diagnostic test is to have the supine patient raise his or her heels off the table to about $15^{\circ}$. In this position, the only active hip flexor is the iliopsoas, and provocative testing as such will elicit pain (15). Our patient provided a history consistent with involvement of her left iliopsoas muscle and on physical examination demonstrated pain with palpation over the inguinal area without or palpable audible snap.

\section{Evaluation}

Initial laboratory studies to rule out infection and radiologic studies can provide further information and rule out other potential causes of anterior hip pain. Plain films may reveal pathologic conditions contributing to the condition such as bony changes and provide information on the positioning of the prosthesis. Computerized tomography (CT) has been useful in demonstrating impingement (3) and a thickened iliopsoas tendon without impingement (2). Fluoroscopically guided arthrography and bursography have demonstrated a bursa extending deep into the pelvis (3). Ultrasonography is useful especially in the setting of groin swelling (6), and in one series of painful snapping hips, documented an immediate temporal correlation between the abnormal tendon motion and the generation of painful symptoms $(11,12)$. Further imaging studies such as technetium bone scanning may help identify a stress fracture, loosening, or infection. MRI has been shown to be the most sensitive and specific method of imaging (15). Interventional procedures have been diagnostically and therapeutically useful.

The CT $(2,7)$, sonographically (5), and in our patient, fluoroscopically guided injection of local anesthetic with corticosteroids into the iliopsoas muscle and tendon provided temporary, complete pain relief and was highly suggestive it was the source of pain. Although there are different techniques of injection described in the literature targeting the bursa and the distal iliopsoas tendon, the experience of this center has been to inject along the iliopsoas muscle. A relative contraindication for distal tendon injection includes having a pre-existing condition of intra-abdominal adhesions. In our patient, due to the presence of her hip prosthesis, plain films and technetium were used as part of her radiologic workup and fluoroscopy was used for the interventional procedure. It is unclear at this time whether sonography or fluoroscopy would provide superior results for this type of procedure and would need further studies.

\section{Management}

Initial non-operative management of iliopsoas tendinitis and bursitis includes rest, stretching and strengthening exercises, nonsteroidal anti-inflammatory medications, and a physical therapy program (8, 15). Anesthetics such as xylocaine or bupivicaine along with a steroid have been adjuncts to a comprehensive rehabilitation program. However the pain relief with this method has been short lived with the eventual need for surgery after symptoms recurred (2-5). In one case series, the duration of pain relief ranged from 2 weeks to 2 years (7).

To date, the successes of surgical outcomes as the definitive treatment have been documented mainly by case reports and case series. After a primary THA, iliopsoas tendinitis has been attributed to impingement of the tendon by the anterior rim of the acetabular component which was confirmed by observation of a frayed tendon in each patient during revision surgery (1). The 2 patients described by Trousdale et al (1) underwent debridement of the tendon and repositioning of the acetabular component and experienced complete pain relief. However, in the absence of radiographic evidence for iliopsoas impingement or malpositioning of the acetabular component, longterm relief was provided by iliopsoas tendon release from the lesser trochanter despite a trial of injected anesthetic and steroids to the muscle (2-5). The literature is limited in long-term outcomes of these surgeries, which are different in technique and approach, tendon release or tendon lengthening, and further study is needed in this area. In one retrospective review of 92 cases of painful snapping hips attributed to the iliopsoas tendon, only $60 \%$ of patients were complication-free that underwent iliopsoas tendon lengthening $(13,14)$.

In our patient, the history, clinical findings, and a positive muscle block were supportive of iliopsoas tendinitis and she failed to have significant long-term pain relief with the conventional treatment of medications, physical therapy, and injection therapy with anesthetic. It was possible that our patient had a 
component of muscle spasm causing her ongoing pain that had not been treated with previous therapies. Therefore, we proceeded with a trial with BTX-A into the iliopsoas muscle with ongoing physical therapy and pain medications.

Botulinum toxin type $\mathrm{A}$ is produced by Clostridium botulinum. The toxin is internalized presynaptically at cholinergic nerve terminals where it inhibits the release of acetylcholine, the neurotransmitter involved in muscle contraction. This chemodenervation manifests as decreased muscle tone, clonus, and muscle overactivity. The effects of BTX-A are evident in 3-10 days and lasts for approximately 3 months as a result of new growth at nerve endings (16). There are recent observations on the anti-nociceptive effects of BTX-A partly explained by inhibition of inflammatory pain by a mechanism not fully established (17).

As part of a single center randomized controlled trial, 7 iliopsoas muscles were injected at 2 sites with 150 units of BTX-A for the treatment of myofascial pain and pain from muscle spasm. BTX-A was found to be more efficacious over conventional steroid injection at the 60-day follow up (18). Both arms of the trial used the injections in combination with a physical therapy program. We used 100 units over 3 sites on the muscle, which is within the range recommended in the spasticity literature for the treatment in this muscle group (19). Further studies are needed to assess the optimal dose, the location, and the number of sites to be injected for this indication.

\section{Significance of ODI and PI-NRS}

For tracking this patient's outcome measures, we evaluated her functional status using the Oswestry Disability Index (ODI) before and after the injection as well as her pain scores on a $0-10$ point pain intensity numerical rating scale (PI-NRS). The ODI remains a valid and worthwhile assessment tool designed to assess pain-related disability in persons with low back pain (20). This is a self-administered outcome measure that takes approximately 5 minutes to complete. Higher scores represent more disability. Clinically significant change in pain scores has been difficult to study due to the subjective experience of pain. The analysis of 10 trials evaluating treatment of chronic pain found that a decrease of 2 points from the baseline pain score is clinically significant (21). During her one-month follow up, the change in her ODI was only 4 percent and she remained in the mild to moderate disability category although she did report a significant decrease in pain from a 7 to 1 and had increased range of motion in left hip flexion. By the 6 months follow up, her ODI was $18 \%$, which now placed her in mild disability, but her pain score increased to 8 which was one point higher than baseline. The conflict in the outcome measure at 6 months may be due to several factors. By 6 months, it is likely that the muscle blocking effect of the BTX-A had worn off and as a result she is experiencing pain that is slightly higher than baseline despite a lower ODI score. The ODI score does account for pain intensity as one of the ten items and may better reflect her global experience with this intervention. The patient may choose to undergo another repeat injection and continue with her rehabilitation program.

\section{Conclusion}

Although iliopsoas tendinitis is a rare cause of groin pain, it should be considered in a patient who has undergone THA after ruling out other etiologies. To date, literature to suggest that surgical management provides long-term pain relief from iliopsoas tendinitis is limited. Non-operative management has not been standardized and requires further investigation.

In summary, treatment with BTX-A to the iliopsoas muscle was effective and well tolerated in providing clinically significant temporary pain relief and reducing the severity of disability in a patient with iliopsoas muscle pain post THA. The use of BTX-A in this case may represent an alternative to the conventional injection of anesthetic and steroids as well as a means to postpone or obviate the need for repeat surgical intervention to the hip. 


\section{References}

1. Trousdale RT, Cabanela ME, Berry DJ. Anterior iliopsoas impingement after total hip arthroplasty. I Arthroplasty 1995; 10:546-549.

2. Della Valle CJ, Rafii M, Jaffe WL. Case Report: Iliopsoas tendinitis after total hip arthroplasty. J Arthroplasty 200; 16:923926.

3. Heaton K, Dorr LD. Technical Note: Surgical release of iliopsoas tendon for groin pain after total hip arthroplasty. J Arthroplasty 2002; 17:779-781.

4. Taher RT, Power RA. Case Report: Iliopsoas tendon dysfunction as a cause of pain after total hip arthroplasty relieved by surgical release. J Arthroplasty 2003; 18:387-388. .

5. Wank R, Miller TT, Shapiro JF. Sonographically guided injection of anesthetic for iliopsoas tendinopathy after total hip arthroplasty. J Clin Ultrasound 2004; 32:354-357.

6. Cheung YM, Gupte CM, Beverly MJ. IIiopsoas bursitis following total hip replacement. Arch Orthop Trauma Surg 2004; 124:720-723.

7. Ala ET, Remy F, Chantelot C, Giraud F, Migaud H, Duquennoy A. Anterior iliopsoas impingement after total hip ar- throplasty: diagnosis and treatment in 9 cases. Rev Chir Orthop Reparatrice Appar Mot 2001; 87:815-819. .

8. Johnston CA, Wiley JP, Lindsay DM, Wiseman DA. Iliopsoas bursitis and tendinitis: a review. Sports Med 1998; 25:271

9. O'Connor. Early recognition of iliopectineal bursitis. Surg Gynecol Obstet 1933; 57:67.

10. Jacobson T, Allen WC. Surgical correction of the snapping iliopsoas tendon. Am J Sports Med 1990; 18:470-474.

11. Allen WC, Cope R. Coxa Saltans: The snapping hip revisited. J Am Acad Orthop Surg 1995; 3:303-308 .

12. Pelsser V, Cardinal E, Hobden R, Benoit A, Lafortune M. Extraarticular snapping hip. Am J Roentgenol 2001; 176:67-73 .

13. Hoskins JS, Burd TA, Allen WC. Surgical correction of internal coxa saltans. Am J of Sports Med 2004; 32:998-1002.

14. Blankenbaker DG, De Smet AA, Keene JS. Sonography of the iliopsoas tendon and injection of the iliopsoas bursa for diagnosis and management of the painful snapping hip. Skeletal Radiol 2006; 35:565-571.
15. Morelli V. Groin injuries and groin pain in athletes: Part 1. Prim Care 2005; 32:163-183.

16. Monnier G, Tatu L, Michel F. Review: New indications for botulinum toxin in rheumatology. Joint Bone Spine 2006; 73:667-671.

17. Borodic GE, Acquadro M, Johnson EA. Botulinum toxin therapy for pain and inflammatory disorders: mechanisms and therapeutic effects. Expert Opin Investg Drugs 2001; 10:1531-1544 .

18. Porta M. A comparative trial of botulinum toxin type $A$ and methylprednisone for the treatment of myofascial pain syndrome and pain from chronic muscle spasm. Pain 2000; 85:101-105 .

19. Spasticity: Etiology, evaluation, management, and the role of Botox A. Muscle Nerve 1997; 6(Suppl):S151 .

20. Fairbank JCT, Pynsent PB. The Oswestry Disability Index Literature Review. Spine 2000; 25:2940-2953 .

21. Farrar JT, Young JP, LaMoreaux L, Werth JL, Poole RM. Clinical importance of changes in chronic pain intensity measured on an 11-point numerical pain rating scale. Pain 2001; 94:149-158. 
